\title{
Oxygen-rich AGB stars with optically thin dust envelopes ${ }^{\star}$
}

\author{
A. M. Heras and S. Hony ${ }^{\star \star}$ \\ Astrophysics Missions Division, Research and Scientific Support Department of ESA, ESTEC/SCI-SA, Postbus 299, \\ 2200 AG Noordwijk, The Netherlands \\ e-mail: aheras@rssd.esa.int
}

Received 2 November 2004 / Accepted 26 April 2005

\begin{abstract}
The dust composition and dynamics of the circumstellar envelopes of oxygen-rich AGB stars with low mass-loss rates $\left(5 \times 10^{-8}-10^{-5} M_{\odot} \mathrm{yr}^{-1}\right)$ have been investigated. We have analyzed the ISO-SWS spectra of twenty-eight oxygen-rich AGB stars with optically thin shells, and modelled the observations with the radiative transfer code DUSTY using the optical constants from laboratory dust analogues. This has allowed us to determine the composition of the dust and the physical conditions at the inner edge of the shell. Moreover, by comparing with $\mathrm{CO}$ observations available in the literature, we have determined the gas-to-dust mass ratios and the mass-loss rates of these sources, and analyzed the wind-driving mechanism. The results show that the small amounts of dust present in these envelopes, characterized by visual optical depths in the $0.03-0.6$ range, are enough to drive the wind by radiation pressure on the grains. In some sources there are indications of circumstellar dust that does not contribute to the wind-driving, and that may distributed in a disk or clumps. Other sources show signs of variable mass-loss rates. A grain mixture in the shell consisting of aluminium oxide, melilite, olivine, spinel and $\mathrm{Mg}_{0.1} \mathrm{Fe}_{0.9} \mathrm{O}$ fit the observed spectra well. From these species, only melilite is required to have a fractional abundance greater than $25 \%$ in all cases. Although spinel reproduces the $13 \mu \mathrm{m}$ feature, the absence of the $16.8 \mu \mathrm{m}$ peak in our SWS spectra casts doubts on this identification. The outcome of the modelling reveals that the olivine content in these CSEs increases with pressure and temperature at the inner edge. Moreover, the aluminium oxide percentage in the dust of the envelopes shows a positive correlation with the gas-to-dust mass ratio. These results, together with the derived dust compositions, are consistent with the thermodynamic dust condensation sequence scenario and its freezing-out due to kinetics. However, the temperatures at the inner edge of the shell are substantially lower than those predicted by theory.
\end{abstract}

Key words. stars: AGB and post-AGB - circumstellar matter - stars: late-type - stars: mass-loss - infrared: stars astrochemistry

\section{Introduction}

AGB stars with low mass-loss rates are of particular interest because of the low density in the dust-forming region of their circumstellar envelope (CSE), which affects the wind driving mechanism and the composition of the dust. Several studies have shown that, below a certain density threshold, radiation pressure on dust grains is insufficient to drive the outflow to the observed values (e.g. Gail 1990; Netzer \& Elitzur 1993). Also, low densities in the outflow prevent the dust condensation sequence from being completed. In general, dust condensation occurs in subsequent stages, where the stages are set by the density and temperature at which the condensation product are stable (e.g. Salpeter 1977; Sedlmayr \& Krüger 1997; Lodders $\&$ Fegley 1999). At low mass-loss rates, dust condensation will

^ Based on observations with ISO, an ESA project with instruments funded by ESA Member States (especially the PI countries: France, Germany, The Netherlands and the UK) and with the participation of ISAS and NASA.

$\star \star$ Present address: Institute for Astronomy, K.U. Leuven, Celestijenlaan 200B, 3001 Heverlee, Belgium. halt at an intermediate stage of the condensation-ladder. This is called "freeze-out". Freeze-out in the wind takes place at a distance from the star where it is cold enough for the next condensate in the sequence to form, but the density is too low for the condensation reaction to occur. In this paper we study CSEs of oxygen-rich AGB stars with small opacities, as observed by the Short Wavelength Spectrometer on board ISO. Our aim is to provide further insight in the driving mechanism of outflows in these sources and to investigate what these objects can teach us about dust formation.

\subsection{Dust formation in stellar outflows}

Dust formation in oxygen-rich outflows is not at all well understood; there are active debates on which components form and in which order. The knowledge of the dust composition in these low mass-loss rate environments provides us with information of the various condensation stages. A quantitative determination of the dust composition as a function of mass-loss rate is an important observational benchmark against which the various existing dust formation scenarios can be tested. 
In principle, dust condensation in the outflows of cool stars can be viewed as a complex network of "chemical" reactions in an expanding and cooling gas. These reactions lead from atoms and molecules, via small clusters of molecules to macroscopic grains. This network is far too complex to treat theoretically and produce useful predictions. Moreover, many of the reactions rates of the chemical and physical interactions are unknown. Two useful approximations are commonly applied to make theoretical predictions of the outcome of dust formation (see Sedlmayr \& Krüger 1997, for an overview).

1. To split the process into two separate steps. The first step deals with the formation of small clusters, called cores or seeds at high-densities and temperatures. The second step involves grain growth, where it is assumed that the seeds are already present. During grain growth only the most abundant, condensable species need to be taken into account, because these will determine the bulk of the dust mass. In contrast, seed nucleation depends on the most stable condensate(s) that can form at high temperature. For example, simple oxides of the relatively rare element $\mathrm{Al}$ or the even less abundant Ti are often thought to be the basis of the seeds in O-rich environs.

2. To assume chemical equilibrium (CE) and local thermodynamic equilibrium (LTE) for the chemical-reaction network. This means that the concentrations of the various species at any point in the outflow are given by the chemical equilibrium value determined by the local density and temperature. In general, this assumes one local temperature for all species set by the radiation field of the star (and the envelope) as the local source of heating. In reality different dust grains will have different internal temperatures depending on their opacity, which will affect the chemical reaction rates. Also hydrodynamical effects are ignored in this approximation. The energy input due to shocks is not taken into account. In the case of the low-density winds that are under study here, the effect of the expansion of the envelope can also invalidate the LTE assumption. If the timescale to reach chemical equilibrium exceeds the cooling timescale due to expansion then $\mathrm{CE}$ will not be reached.

Using these assumption several authors have made predictions for the types of dust that condense under astrophysically relevant pressures and temperatures (e.g. Salpeter 1977; Tielens 1990; Sedlmayr \& Krüger 1997; Lodders \& Fegley 1999). These show that aluminium-oxide is the first astrophysically relevant species to condense ( $T \sim 1400 \mathrm{~K})$. At lower temperature this is transformed into spinel $\left(\mathrm{MgAl}_{2} \mathrm{O}_{4}, T \sim 1300 \mathrm{~K}\right)$ and subsequently into melilite $\left(\mathrm{Ca}_{2} \mathrm{Al}_{2} \mathrm{SiO}_{7}\right)$. The bulk of the $\mathrm{Si}$ and $\mathrm{Mg}$ condenses at lower temperature into silicates.

\subsection{Dust driven winds}

On the upper AGB stars experience substantial mass-loss through a dust-driven wind. In essence, this occurs in the following way. At a few stellar radii from the star, dust particles form. These particles efficiently absorb the momentum of the photons of the stellar radiation field, causing them to experience an outward force. Due to the high luminosity of the star the outward force exceeds the inward directed gravitational force and the dust particles are accelerated outward. As the dust grains move outwards they collide with the gas and transfer momentum to the gas. This accelerates the gas away from the star and an outflow is the result.

In order for this process to occur, several conditions need to be fulfilled. The most important one in the framework of this paper is the abundant formation of dust. Dust formation is efficient only in a limited domain of pressure and temperature. In the stellar surroundings both the density and pressure will, in general, decrease with distance from the star. If the density is too low at the location where the temperature is low enough to allow dust condensation, dust will not form in the outflow. The type of stars under consideration is characterized by having optically thin envelopes and a similar temperature profile $(T \propto$ $r^{-0.5}$ ) which implies that the determining factor is the density at a few stellar radii. The dust formation has been evaluated numerically by several authors (e.g. Sedlmayr \& Dominik 1995; Gail 1990). It has been found that the lower limit for efficient dust formation, and therefore dust driving of the outflow, occurs within the range of mass-loss rates in our sample $(\dot{M} \sim$ $10^{-6} M_{\odot} \mathrm{yr}^{-1}$, Gail 1990). According to these studies, at lower mass-loss rates insufficient dust is formed to establish a dust driven outflow.

\subsection{Previous studies of oxygen-rich CSES}

The characterization of the dust in the CSEs has greatly advanced through the study of the spectral features in the midinfrared wavelength range. IRAS/LRS provided a large spectral database to make individual and statistical analysis of the shells around AGB stars. Onaka et al. (1989) fitted successfully the IRAS LRS spectra of 109 Mira variables, by assuming aluminium oxide and amorphous magnesium silicate, and dust temperatures of $200-500 \mathrm{~K}$ at the inner boundary of the dust shell. These authors concluded that these low temperatures could not be understood in the framework of homogeneous nucleation theories, and proposed that mantle growth on pre-existing aluminium grains was the major process of silicate formation. Sloan \& Price (1998), through the classification of the IRAS/LRS spectra of 635 oxygen rich variables, showed the existence of a smoothly varying sequence of spectral shapes, from the broad features to the typical silicate feature, that they called the silicate dust sequence. The lower silicate dust sequence resulted from amorphous alumina grains, while the upper part was associated with amorphous silicates. These authors also found dependencies of the dust emission on the properties of the central star. For example, Mira variables with symmetric light curves generally showed broad alumina emission, and those with more asymmetric light curves presented classic silicate emission. Sloan \& Price (1998) proposed that differences in the photospheric $\mathrm{C} / \mathrm{O}$ ratio could be the reason. Egan \& Sloan (2001) made radiative transfer models of the LRS spectra of oxygen rich circumstellar shells, and concluded that optically thin shells dominated by amorphous silicate grains reproduce the classic narrow silicate feature at $10 \mu \mathrm{m}$. Spectra with broad, low-contrast emission features peaking at 
wavelengths longer than $\sim 11 \mu \mathrm{m}$, originated from optically thin shells composed of amorphous alumina. Speck et al. (2000) analyzed UKIRT CGS3 spectra of 80 oxygen-rich AGB stars and found that, while the classic silicate feature was identical for AGB star and red supergiants, the broad features observed were different. As a consequence, they suggested different dust evolutionary paths. While the dust features around the Mira stars could be fit with a combination of aluminium oxide and magnesium silicate, the dust features in the supergiants were fit with a combination of $\mathrm{Ca}-\mathrm{Al}$-rich silicate and aluminium oxide.

The Infrared Space Observatory (ISO) observed over 900 objects with the Short Wavelength Spectrometer (SWS, de Graauw et al. 1996) in full grating scan mode $(2.38-45.2 \mu \mathrm{m})$. The SWS was better suited for the study of dust than previous instruments because of its higher spectral resolution and its larger wavelength range coverage. Thanks to these characteristics, better and more detailed comparisons with laboratory spectral features became feasible, and therefore a better discrimination of dust species. In contrast with the LRS, the SWS also observed the stellar photosphere in sources with optically thin CSEs, which provides essential information on the source type and makes it possible to correlate molecular lines and dust properties.

Kraemer et al. (2002) classified the SWS spectra in seven groups. Relevant to this study is group 2, that comprises stars with dust, but whose SEDs are dominated by the stellar photosphere. The 2.SE subgroup corresponds to the oxygen-rich dust emission, which is sub-classified as 2.SEa (broad emission feature peaking at $12 \mu \mathrm{m}$ ), 2.SEb (spectra with a $10 \mu \mathrm{m}$ dust feature associated with silicates, also a secondary peak of the emission at $11 \mu \mathrm{m}$ and often the $13 \mu \mathrm{m}$ feature as well), 2.SEc (strong silicate emission features with peaks at 10 and $18 \mu \mathrm{m})$. This classification was well correlated with the Sloan $\&$ Price (1998) silicate dust sequence. Cami (2002) studied the SWS spectra of twenty-four AGB stars with low mass-loss rates. This author modelled the observed molecular bands and made a qualitative analysis of the composition of the dust in the shells, which showed that low mass-loss rate stars do not produce silicates, but large amounts of $\mathrm{Al}_{2} \mathrm{O}_{3}$, spinel and $\mathrm{MgFeO}$. Based on these results, Cami (2002) suggested a correlation of the different spectral appearance of the SWS spectra with the silicate dust condensation sequence. In another study of the SWS spectra of low mass-loss AGB stars that showed the broad emission feature, Posch et al. (2002) postulated the existence of a distinct class of circumstellar shells, in which the dominant components of the dust are oxides instead of silicates. These authors suggested that aluminium, magnesium and iron oxides represent the first spectroscopically detectable dust species to condense in oxygen rich CSEs.

\section{Observations}

We obtained the data used in this study by querying the ISO archive for observations made with the SWS instrument, in which the SWS01 observing mode was used. This mode covers the entire $2.38-45.2 \mu \mathrm{m}$ SWS spectral range at spectral resolutions of 1000 to 200 (depending on observation and wavelength). From the observations found in the archive, we selected those whose targets fulfilled the following criteria:

1. Giants with late type spectral class.

2. Oxygen-rich variable stars.

3. Classified as 2.SEa or 2.SEb in the classification scheme of the SWS spectra made by Kraemer et al. (2002). For comparison, we took two stars that were classified as $2 . \mathrm{SEc}$, that is, having strong silicate emission features that peak at 10 and $18 \mu \mathrm{m}$.

4. Mass-loss rate and gas expansion velocity derived from $\mathrm{CO}$ radio lines were available in the literature.

5. Sufficient signal-to-noise ratio in the SWS spectrum to clearly distinguish the solid state features.

The resulting list of sources is shown in Table 1. The second column gives the ISO TDT number which uniquely identifies the observation in the ISO archive. Columns three to six list the spectral classification, the variability type, the period and the class by Kraemer et al. (2002), respectively. The next columns display the parameters of the CSE derived from CO radio line emission measurements found in the literature. The distance to the source, $D_{\mathrm{CO}}$, is shown in the sixth column, and the gas mass-loss rate, $\dot{M}$, gas expansion velocity, $v_{\mathrm{e}}$, photodissociation radius, $R_{\mathrm{e}}$, and the literature reference are displayed in the last four columns. A small number of objects show CO line profiles that can be divided into two components, a narrow velocity component $\left(<5 \mathrm{~km} \mathrm{~s}^{-1}\right)$ on a broader plateau. It has been proposed that the narrow component is associated with highly variable gas expansion velocities (Knapp et al. 1998), complicated geometries (Kahane \& Jura 1996) or reservoirs of orbiting gas (Jura \& Kahane 1999). An asterisk attached to the reference number indicates that the expansion velocity and mass-loss rate are derived from this $\mathrm{CO}$ narrow line component.

The data were extracted from the ISO archive and reduced with the SWS Interactive Analysis (IA3) software (e.g., Lahuis et al. 1997), using the OLP V10.1 calibration files and algorithms. We flat fielded the Auto-Analysis result, that is, we aligned the flux profiles of detectors and scans using a fitted polynomial as reference level. We eliminated those points with a deviation from the mean flux value in fixed size bins greater than $3 \sigma$. We also interactively removed segments of the spectra in which a detector exhibited anomalous behavior or where the signal abruptly changed. The next step was to rebin the interactively processed spectra to the instrumental resolution determined by the observing mode. Defringing was applied when required. The SWS spectra are subdivided in wavelength intervals by the manner in which the spectrometer obtained the measurement. These segments, labeled AOT (Astronomical Observation Template) bands, are instrumentally defined by aperture, spectral order, filter band pass and detector type. Due to several instrumental factors (e.g. inaccurate pointing, memory effects, problematic dark current subtraction, different flux calibration accuracy), jumps between AOT bands appear in some SWS spectra. We carefully corrected for these jumps through visual inspection, by shifting the problematic AOT band or by modifying its slope. We have decided on the best correction method for each case, by looking at 
Table 1. Objects parameters and CO data.

\begin{tabular}{|c|c|c|c|c|c|c|c|c|c|c|}
\hline Source & $\begin{array}{c}\text { ISO TDT } \\
\text { number }\end{array}$ & $\begin{array}{c}\text { Spectral } \\
\text { class }\end{array}$ & $\begin{array}{c}\text { Variability } \\
\text { type }\end{array}$ & $\begin{array}{c}\text { Period }^{a} \\
{[\mathrm{~d}]}\end{array}$ & $\begin{array}{c}\text { Kraemer }^{b} \\
\text { class }\end{array}$ & $\begin{array}{l}D_{\mathrm{CO}} \\
{[\mathrm{pc}]}\end{array}$ & $\begin{array}{c}\dot{M}\left(\times 10^{-7}\right) \\
{\left[M_{\odot} \mathrm{yr}^{-1}\right]}\end{array}$ & $\begin{array}{c}v_{\mathrm{e}} \\
{\left[\mathrm{km} \mathrm{s}^{-1}\right]}\end{array}$ & $\begin{array}{c}R_{\mathrm{e}}\left(\times 10^{16}\right) \\
{[\mathrm{cm}]}\end{array}$ & $\overline{\operatorname{Ref}^{c}}$ \\
\hline \multirow[t]{2}{*}{ T Cas } & 40201208 & M7e & Mira & 444.83 & 2.SEa & 290 & 5.1 & 7.2 & 5.4 & 2 \\
\hline & & & & & & 350 & 11.0 & 10.5 & 16.0 & 3 \\
\hline CE And & 80104817 & M5 & $\mathrm{Lb}$ & & 2.SEc & 740 & 5.0 & 10.5 & 2.5 & 1 \\
\hline \multirow[t]{3}{*}{ SV Psc } & 80501620 & M5 & $\mathrm{SRb}$ & 102.00 & 2.SEb & 380 & 3.0 & 9.5 & 1.9 & 1 \\
\hline & & & & & & & 0.3 & 1.5 & 1.0 & $1 *$ \\
\hline & & & & & & 405 & 10.7 & 11.0 & 5.18 & 5 \\
\hline \multirow[t]{6}{*}{$o$ Cet } & 45101201 & M7IIIe & Mira & 331.96 & 2.SEc & 110 & 5.0 & 5.6 & 9.4 & 2 \\
\hline & & & & & & 100 & 3.6 & 4.8 & 14.0 & 3 \\
\hline & & & & & & 128 & 4.4 & 6.7 & 3.8 & 4 \\
\hline & & & & & & & 0.94 & 2.4 & 2.4 & $4^{*}$ \\
\hline & & & & & & 128 & 8.27 & 7.0 & 5.33 & 5 \\
\hline & & & & & & & 1.17 & 1.5 & 3.17 & $5^{*}$ \\
\hline RV Cam & 86202101 & M4II-III & $\mathrm{SRb}$ & 101.00 & 2.SEb & 350 & 2.5 & 5.8 & 2.0 & 1 \\
\hline \multirow[t]{2}{*}{ Y UMa } & 60200502 & M7II-III & $\mathrm{SRb}$ & 168.00 & 2.SEa & 220 & 1.5 & 4.8 & 1.7 & 1 \\
\hline & & & & & & 313 & 1.7 & 5.4 & & 4 \\
\hline \multirow[t]{6}{*}{ R Hya } & 08200502 & M7IIIe & Mira & 388.87 & 2.SEa & 130 & 1.4 & 8.0 & 2.7 & 2 \\
\hline & & & & & & 110 & 4.2 & 10.5 & 10.0 & 3 \\
\hline & & & & & & 200 & 4.7 & 11.0 & 3.3 & 4 \\
\hline & & & & & & & 0.73 & 5.0 & 6.0 & $4^{*}$ \\
\hline & & & & & & 125 & 5.19 & 10.0 & 3.53 & 5 \\
\hline & & & & & & & 1.34 & 4.5 & 2.21 & $5^{*}$ \\
\hline \multirow[t]{3}{*}{ S Vir } & 25302224 & M7IIIe & Mira & 375.10 & 2.SEa & 410 & 4.1 & 9.2 & 5.0 & 2 \\
\hline & & & & & & 450 & 0.56 & 4.4 & 5.6 & 3 \\
\hline & & & & & & 420 & 0.8 & 5.2 & & 4 \\
\hline \multirow[t]{3}{*}{ W Hya } & 08902004 & M8e & SRa & 361.00 & 2.SEa & 65 & 0.7 & 6.5 & 1.0 & 1 \\
\hline & & & & & & 80 & 1.8 & 8.0 & 7.5 & 3 \\
\hline & & & & & & 115 & 1.7 & 8.1 & & 4 \\
\hline$\theta$ Aps & 07901809 & M6.5III: & $\mathrm{SRb}$ & 119.00 & 2.SEb & 110 & 0.40 & 4.5 & 0.8 & 1 \\
\hline RX Boo & 08201905 & M7.5 & $\mathrm{SRb}$ & 340.00 & 2.SEa & 110 & 5.0 & 9.3 & 2.6 & 1 \\
\hline RV Boo & & & & & & & 0.3 & 2.3 & 0.8 & $1^{*}$ \\
\hline g Her & 80000104 & M6III & $\mathrm{SRb}$ & 89.200 & 2.SEa & 100 & 1.0 & 8.4 & 1.3 & 1 \\
\hline \multirow[t]{2}{*}{ X Oph } & 47201847 & M5e-M9e & Mira & 328.85 & 2.SEa & 270 & 0.45 & 6.1 & 4.2 & 3 \\
\hline & & & & & & 186 & 2.5 & 6.0 & 2.83 & 5 \\
\hline FI Lyr & 82700735 & M6 & $\mathrm{SRb}$ & 146.00 & 2.SEa & 880 & 21. & 12.3 & 12. & 2 \\
\hline \multirow[t]{2}{*}{ S Pav } & 14401702 & M7IIe & $\mathrm{SRa}$ & 380.86 & 2.SEa & 150 & 0.8 & 9.0 & 1.1 & 1 \\
\hline & & & & & & 345 & 4.98 & 9.0 & 3.59 & 5 \\
\hline RR Aql & 53400809 & M7e & Mira & 394.78 & 2.SEc & 420 & 9.1 & 8.4 & 7.7 & 2 \\
\hline V1943 Sgr & 85700514 & M7III... & $\mathrm{Lb}$ & & 2.SEa & 150 & 1.3 & 5.4 & 1.4 & 1 \\
\hline V584 Aql & 73200811 & M8 & $\mathrm{Lb}$ & & 2.SEb & 390 & 0.5 & 2.2 & 1.2 & 1 \\
\hline X Pav & 14401801 & M6/M7III & $\mathrm{SRb}$ & 199.19 & 2.SEb & 270 & 7.3 & 11.1 & 6.5 & 2 \\
\hline T Mic & 14401129 & M7III... & $\mathrm{SRb}$ & 347.00 & 2.SEa & 130 & 0.8 & 4.8 & 1.2 & 1 \\
\hline \multirow[t]{2}{*}{ T Cep } & 26300141 & M7IIIe & Mira & 388.14 & 2.SEa & 140 & 1.4 & 9.7 & 3.4 & 2 \\
\hline & & & & & & 220 & 0.99 & 4.8 & 7.2 & 3 \\
\hline \multirow[t]{6}{*}{ EP Aqr } & 38600922 & M8IIIvar & $\mathrm{SRb}$ & 55.000 & 2.SEb & 140 & 5.0 & 9.2 & 2.5 & 1 \\
\hline & & & & & & & 0.3 & 1.0 & 1.1 & $1 *$ \\
\hline & & & & & & 135 & 2.3 & 10.8 & 2.3 & 4 \\
\hline & & & & & & & 0.17 & 1.4 & 1.1 & $4^{*}$ \\
\hline & & & & & & 135 & 7.91 & 11.0 & 4.34 & 5 \\
\hline & & & & & & & 0.48 & 1.0 & 2.23 & $5^{*}$ \\
\hline SV Peg & 74500605 & M7 & $\mathrm{SRb}$ & 144.60 & 2.SEa & 190 & 3.0 & 7.5 & 2.1 & 1 \\
\hline \multirow[t]{2}{*}{ DZ Aqr } & 53500647 & M... & SR: & & 2.SEb & 840 & 9.7 & 8.2 & 8.7 & 2 \\
\hline & & & & & & 285 & 5.75 & 7.0 & 4.32 & 5 \\
\hline RX Lac & 78200427 & М... & $\mathrm{SRb}$ & 650.00 & 2.SEa & 250 & 0.8 & 2.2 & 1.6 & 1 \\
\hline R Peg & 41500123 & M7e & Mira & 378.10 & 2.SEa & 380 & 1.2 & 8.3 & 6.1 & 3 \\
\hline & & & & & & 350 & 4.06 & 5.5 & 3.89 & 5 \\
\hline
\end{tabular}

${ }^{a}$ Combined General Catalogue of Variable Stars (Kholopov et al. 1998), except for the values of $o$ Cet and RR Aql, taken from Barthès et al. (1999).

${ }^{b}$ Kraemer et al. (2002).

${ }^{c}$ The references of the measurements are: (1) Olofsson et al. (2002); (2) Loup et al. (1993); (3) Young (1995); (4) Knapp et al. (1998); (5) Winters et al. (2003). The asterisk indicates that these values have been derived from the narrow feature of a double-component CO line. 
the general spectral shape and by checking the overlap areas between AOT bands.

\section{Model}

We have performed a parametrised study that allows us to have a better understanding of the physical and chemical conditions that prevail in the outflows of the stars in our sample. Such modelling cannot, nor is intended to, model all the physical and chemical processes involved in the formation of the dust or the driving of the outflow. As a consequence, the details of the outcome may be questionable because of the simplifications made. However, the model is valid to construct a first-order overall picture and, as a first descriptive step, can provide useful input and constraints to future self-consistent models.

We have used the spherical case of the 1-D code DUSTY (Ivezić et al. 1999) to model the radiative transfer through the dust shell and to reproduce the observed ISO-SWS spectra. In the next sub-sections, we describe and discuss the input parameter values adopted in the modelling. The resolution of the grid for these input parameters results from a compromise between the accuracy of the model fit and the required computational time.

\subsection{Model input}

\subsubsection{Stellar radiation}

Due to the scale invariance in the radiative transfer problem, the only relevant property of the external radiation is its spectral shape (Ivezić et al. 1999). We have taken a composed spectrum for the stars in our sample. For wavelengths shorter than $2.6 \mu \mathrm{m}$, a black body with a $T_{\text {eff }}$ equal to $2800 \mathrm{~K}$ has been assumed. For $2.6 \mu \mathrm{m} \leq \lambda \leq 8.7 \mu \mathrm{m}$, we have adopted the observed photospheric ISO-SWS spectrum, and at wavelengths longer than $8.7 \mu \mathrm{m}$ we have applied a Rayleigh-Jeans extrapolation. For a few stars, the assumed $T_{\text {eff }}=2800 \mathrm{~K}$ proved to be too high for an accurate fit, therefore a $T_{\text {eff }}=2200 \mathrm{~K}$ was taken instead. Table 2 displays the values adopted for each object.

\subsubsection{Dust properties}

A standard MRN distribution with the modification proposed by Kim et al. (1994) has been adopted for the grain size of all sources, $n(a) \propto a^{-q} \mathrm{e}^{-a / a_{0}}$, with the parameters $q$ equal to 3.5 , $a(\mathrm{~min})$ equal to $0.005 \mu \mathrm{m}$ and $a_{0}$ equal to $0.2 \mu \mathrm{m}$. We have chosen the option provided by DUSTY to derive the density distribution of the dust from a full dynamics calculation of a typical wind driven by radiation pressure on the dust grains. A determinant input parameter is the dust temperature at the shell inner boundary, $T_{\mathrm{d}}$. The code only allows the user to specify a temperature value for all dust components, although, in reality, the temperatures of each grain type could be rather different.

Concerning the composition of the dust, DUSTY supports grain mixtures by simulating a single type grain constructed from a given average. We have selected the materials of the dust shell by relying on previous literature studies and by experimenting with the optical constants available. In particular, the data used in this work have been retrieved from the Database of Optical Constants of the Laboratory Astrophysics Group of the AIU Jena ${ }^{1}$. The following dust grain types have been considered in the modelling:

- Amorphous aluminium oxide $\mathrm{Al}_{2} \mathrm{O}_{3}$ (Begemann et al. 1997). As explained in Sect. $1, \mathrm{Al}_{2} \mathrm{O}_{3}$ is the first condensate in an expanding oxygen-rich cooling-gas according to thermodynamic equilibrium condensation calculations. Onaka et al. (1989) were the first to reproduce the broad $12 \mu \mathrm{m}$ feature observed in IRAS spectra of AGB stars using aluminium oxide grains. Since then, other studies have successfully used $\mathrm{Al}_{2} \mathrm{O}_{3}$ to simulate the observed spectra (e.g., Speck et al. 2000; Egan \& Sloan 2001; Posch et al. 2002), or as a initial condensate for theoretical dust formation models (e.g., Sogawa \& Kozasa 1999).

- Melilite $\left(\mathrm{Ca}_{2} \mathrm{Al}_{2} \mathrm{SiO}_{7}\right)$ was proposed by Mutschke et al. (1998) as one of the main components to explain the IRAS LRS spectra of two Mira stars. Speck et al. (2000) also used melilite to reproduce the observed spectra of a group of supergiants in the $8-13 \mu \mathrm{m}$ region. We have chosen this mineral because its optical constants are the most suitable to fit simultaneously the $10 \mu \mathrm{m}$ and $19-20 \mu \mathrm{m}$ features observed in most of the stars in our sample. The olivines and pyroxenes in the AIU Jena database reproduce worse our spectra, and this finding is consistent with the conclusions by Mutschke et al. (1998).

- Spinel $\left(\mathrm{MgAl}_{2} \mathrm{O}_{4}\right)$ has been suggested by Posch et al. (1999) as carrier of the $13 \mu \mathrm{m}$ feature observed frequently in the spectra of optically thin dust envelopes of oxygenrich AGB stars. Currently this is the best accepted candidate, although no consensus has been reached yet.

- $\mathrm{Mg}_{0.1} \mathrm{Fe}_{0.9} \mathrm{O}$ was identified by Cami (2002) and Posch et al. (2002) as the carrier of the 19.5 dust emission feature that was discovered in the SWS spectra of AGB stars with optically thin shells.

- Olivine $\left(\mathrm{Mg}_{0.8} \mathrm{Fe}_{1.2} \mathrm{SiO}_{4}\right)$. We have chosen this particular analogue as representative of the typical silicates that are observed in AGB stars, characterized by prominent $9.7 \mu \mathrm{m}$ and $18 \mu \mathrm{m}$ features. These peaks appear in the stars of our sample with a wide range of strengths.

\subsection{Fitting the model output to the observed spectra}

For each star we have assumed an input stellar radiation spectra (see Sect. 3.1.1). Then we have constructed a grid of models by running DUSTY with different values of the input parameters: Dust temperature at the inner boundary, optical depth, shell thickness relative to inner radius, dust chemical composition (given by fractional abundances of the components listed above). We have selected the best model through eyeball comparison between the output spectral shape and the observed spectrum. In some cases, to improve the quality of the fit required to build a finer grid of models around a certain input parameter set. The fits are shown in Fig. 1, where it can be seen that the SWS spectra and the output of the model agree rather

\footnotetext{
${ }^{1}$ http://www .astro.uni-jena.de/Laboratory/Database/ databases.html
} 


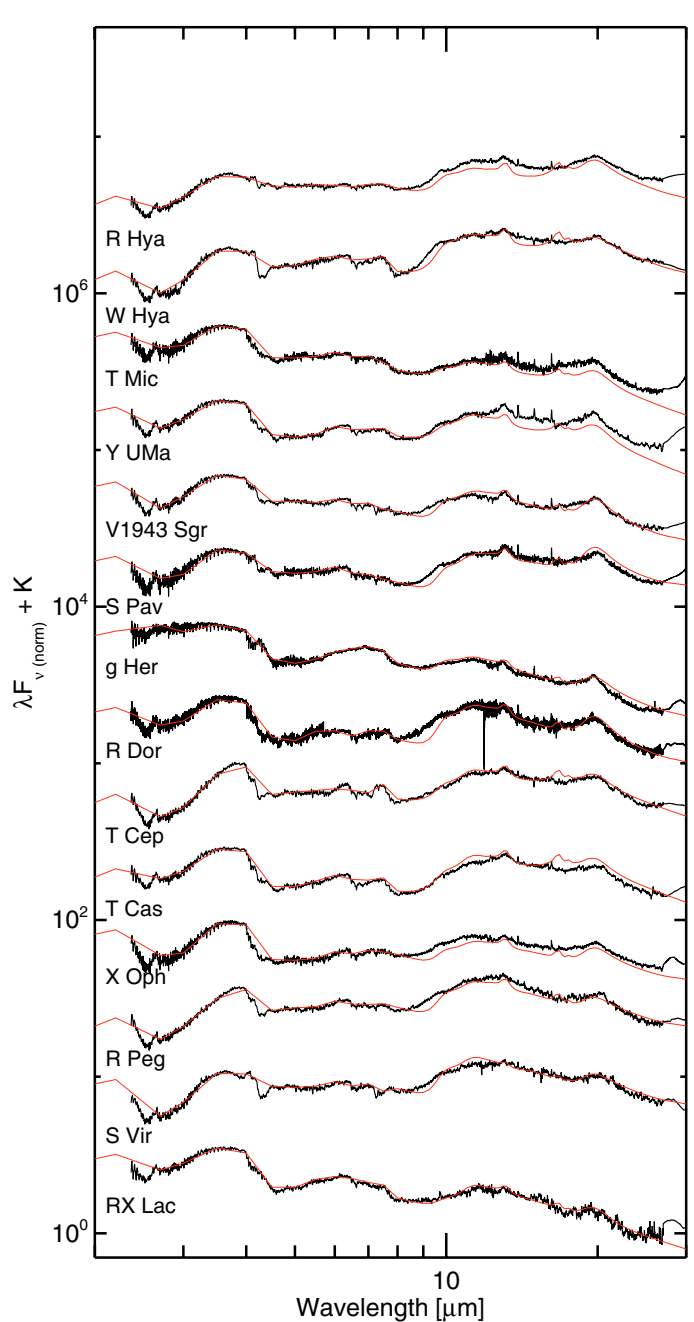

Fig. 1. SWS stellar spectra, in black, and model fit, in grey.

well. In some stars, however, there is a small discrepancy in the wavelength range between 8 and $9 \mu \mathrm{m}$, and the $16.8 \mu \mathrm{m}$ feature that pertains to the laboratory analogue of spinel does not appear in our observations. SWS band $4(28-45.2 \mu \mathrm{m})$, not shown in Fig. 1, has been taken into account in the fitting procedure, although with care, because of the larger calibration uncertainties involved in that wavelength range. The absolute flux calibration error in band 4 is around 20\%, in many sources the signal to noise ratio is very low, and in some cases an unidentified broad feature appears around $28 \mu \mathrm{m}$, which has tentatively been linked to crystalline silicates (Sloan et al. 2003).

DUSTY provides as output the inner radius of the shell, the outer radius, the dust temperature at the envelope's outer edge and, most important for our study, the mass-loss rate and the terminal outflow velocity. The mass-loss rate scales in proportion to the luminosity of the star as $L^{3 / 4}$ and to the gas-to-dust mass ratio as $r_{\mathrm{gd}}^{1 / 2}$. The terminal velocity scales in proportion to $L^{1 / 4}$ and $r_{\mathrm{gd}}^{-1 / 2}$.

The thickness of the shell is the least accurate output parameter. In order to constrain its value (e.g. 100 or 1000 times the inner radius), data at longer wavelengths than those covered by the SWS are necessary. For this purpose, we took IRAS photometry at 60 and $100 \mu \mathrm{m}$, but this approach is not valid for all

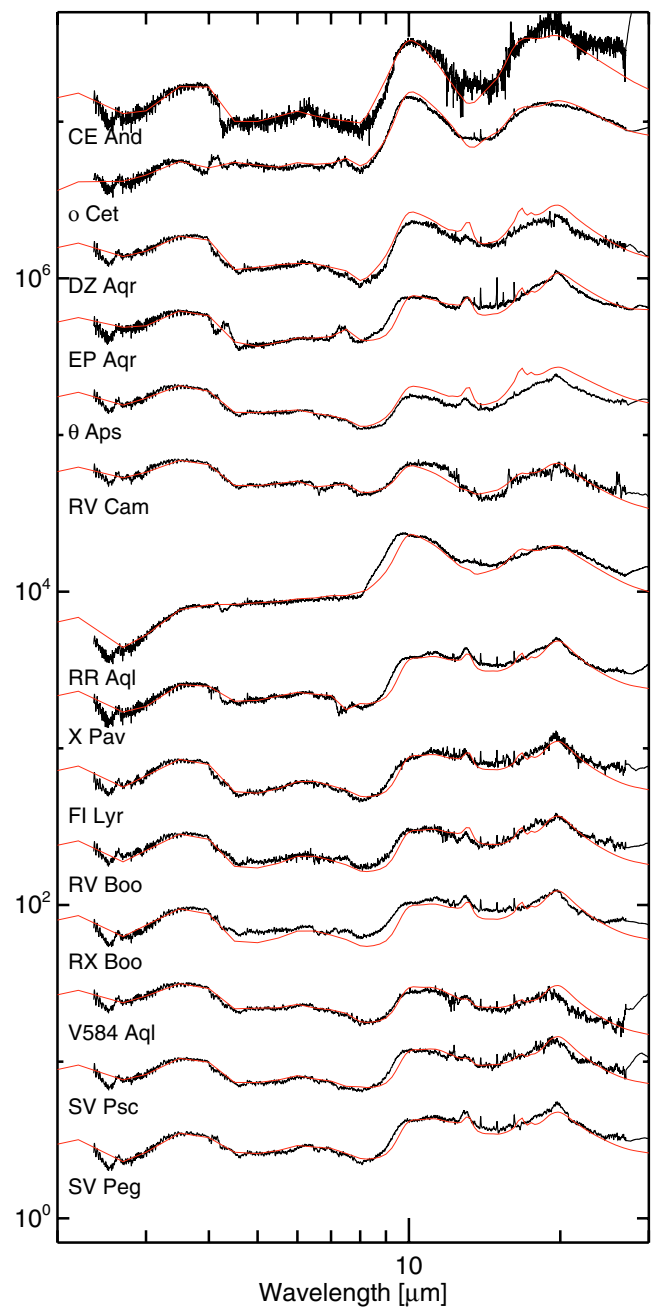

stars. In an important fraction of objects, the IRAS fluxes at 12 and $25 \mu \mathrm{m}$ are significantly different from the ISO-SWS fluxes, because of stellar variability and opacity changes in the dust shell. To assume that the IRAS 60 and $100 \mu \mathrm{m}$ fluxes were good extrapolations of the ISO-SWS fluxes would have been incorrect in these cases. We could only determine a lower limit for the shell thickness, which we took equal to 100 times the inner radius. Fortunately, this does not have a significant impact on the other model output parameters, because for the low mass-loss rates of our sample the density is very low in the outer layers and there the terminal velocity has already reached its asymptotic value.

\section{Results}

Table 2 lists the input and output model parameters and Fig. 2 shows the dust compositions that provide the best model fits. The dust temperatures at the inner boundary are in the range 400-700 K, which corresponds to distances of around 30 and $10 R_{*}$, respectively. Since the model does not allow us to specify different temperatures for the various grain materials, and the silicate features are the most temperature sensitive, the values obtained through the fits are mainly determined by the 
Table 2. Model parameters.

\begin{tabular}{|c|c|c|c|c|c|c|c|c|c|c|c|c|c|c|c|c|}
\hline \multirow[b]{3}{*}{ Source } & & & & & \multicolumn{12}{|c|}{ Output parameters } \\
\hline & \multicolumn{4}{|c|}{ Input parameters } & \multicolumn{7}{|c|}{$r_{\mathrm{gd}}^{a}$} & \multicolumn{5}{|c|}{$\dot{M}\left[\times 10^{-7} M_{\odot} \mathrm{yr}^{-1}\right]^{a}$} \\
\hline & $\begin{array}{c}T_{*} \\
{[\mathrm{~K}]}\end{array}$ & $\begin{array}{c}T_{\mathrm{d}} \\
{[\mathrm{K}]}\end{array}$ & $\tau_{\mathrm{V}}$ & $\begin{array}{c}D \\
{[\mathrm{pc}]}\end{array}$ & $\begin{array}{c}R_{\mathrm{i}} \\
{\left[R_{*}\right]}\end{array}$ & $\begin{array}{c}v_{\mathrm{e}} \\
{\left[\mathrm{km} \mathrm{s}^{-1}\right]}\end{array}$ & (1) & (2) & (3) & (4) & (5) & (1) & (2) & (3) & (4) & (5) \\
\hline T Cas & 2200 & 500 & 0.18 & 350 & 12.4 & 6.6 & & 166.5 & 78.3 & & & & 8.5 & 5.8 & & \\
\hline CE And & 2800 & 600 & 0.19 & 740 & 22.3 & 5.6 & 57.8 & & & & & 2.1 & & & & \\
\hline SV Psc & 2800 & 500 & 0.10 & 380 & 26.3 & 4.7 & 48.4 & & & & 36.1 & 1.6 & & & & 1.4 \\
\hline$o$ Cet & 2200 & 600 & 0.64 & 128 & 9.7 & 8.3 & & 438.2 & 596.5 & 306.1 & 280.5 & & 25.4 & 29.7 & 21.3 & 20.4 \\
\hline RV Cam & 2800 & 500 & 0.06 & 332 & 27.4 & 3.9 & 91.8 & & & & & 1.3 & & & & \\
\hline R Dor & 2800 & 600 & 0.07 & 62 & 17.5 & 6.2 & 212.2 & & & & & 4.1 & & & & \\
\hline Y UMa & 2800 & 600 & 0.05 & 312 & 19.5 & 5.8 & 294.6 & & & 232.8 & & 4.0 & & & 3.6 & \\
\hline R Hya & 2200 & 400 & 0.20 & 617 & 17.7 & 9.5 & & 283.5 & 164.6 & 150.0 & 181.5 & & 73.4 & 55.9 & 53.4 & 58.7 \\
\hline S Vir & 2800 & 500 & 0.12 & 328 & 18.1 & 5.5 & & 70.2 & 307.0 & 219.8 & & & 2.3 & 4.8 & 4.1 & \\
\hline W Hya & 2200 & 500 & 0.30 & 114 & 11.5 & 6.8 & 215.8 & & 142.5 & 139.0 & & 10.9 & & 8.8 & 8.7 & \\
\hline$\theta$ Aps & 2800 & 400 & 0.10 & 100 & 35.2 & 3.8 & 143.2 & & & & & 2.9 & & & & \\
\hline RX Boo & 2800 & 500 & 0.10 & 155 & 26.0 & 5.5 & 69.7 & & & 65.4 & & 3.3 & & & 3.2 & \\
\hline RV Boo & 2800 & 500 & 0.10 & 393 & 26.0 & 5.6 & 126.7 & & & & & 4.6 & & & & \\
\hline g Her & 2800 & 600 & 0.03 & 110 & 17.2 & 4.2 & 50.3 & & & & & 0.5 & & & & \\
\hline X Oph & 2800 & 400 & 0.05 & 270 & 28.7 & 4.9 & & & 130.6 & & 135.0 & & & 4.7 & & 4.7 \\
\hline FI Lyr & 2800 & 500 & 0.10 & 641 & 26.0 & 6.0 & & 47.7 & & & & & 3.6 & & & \\
\hline S Pav & 2800 & 400 & 0.07 & 343 & 31.9 & 6.4 & 101.3 & & & & 101.3 & 10.5 & & & & 10.5 \\
\hline RR Aql & 2800 & 700 & 0.28 & 403 & 15.6 & 9.7 & & 264.4 & & & & & 14.6 & & & \\
\hline V1943 Sgr & 2800 & 500 & 0.05 & 549 & 22.7 & 8.3 & 467.0 & & & & & 23.4 & & & & \\
\hline V584 Aql & 2800 & 600 & 0.07 & 390 & 20.0 & 5.0 & 1038.3 & & & & & 5.3 & & & & \\
\hline X Pav & 2800 & 500 & 0.14 & 609 & 26.0 & 9.9 & & 158.2 & & & & & 29.3 & & & \\
\hline T Mic & 2800 & 500 & 0.03 & 290 & 25.3 & 5.6 & 277.1 & & & & & 5.7 & & & & \\
\hline T Cep & 2200 & 500 & 0.20 & 210 & 12.4 & 6.0 & & 77.2 & 315.2 & & & & 4.3 & 8.8 & & \\
\hline EP Aqr & 2800 & 400 & 0.12 & 135 & 34.9 & 4.1 & 40.3 & & & 29.3 & 28.2 & 2.1 & & & 1.8 & 1.8 \\
\hline SV Peg & 2800 & 500 & 0.12 & 196 & 23.8 & 4.6 & 75.7 & & & & & 1.9 & & & & \\
\hline DZ Aqr & 2800 & 600 & 0.14 & 284 & 20.7 & 5.3 & & 83.5 & & & 114.6 & & 2.0 & & & 2.3 \\
\hline RX Lac & 2800 & 600 & 0.03 & 250 & 17.3 & 4.4 & 795.7 & & & & & 2.3 & & & & \\
\hline R Peg & 2200 & 500 & 0.28 & 349 & 9.4 & 5.5 & & & 87.7 & & 199.7 & & & 3.2 & & 4.8 \\
\hline
\end{tabular}

${ }^{a}$ Calculated with the CO velocity measurements from: (1) Olofsson et al. (2002); (2) Loup et al. (1993); (3) Young (1995); (4) Knapp et al. (1998); (5) Winters et al. (2003).

melilite and the olivine. We have run several tests, composing modelled spectra in which the temperature of the aluminium oxide was taken as $1000 \mathrm{~K}$. The profiles obtained in this way showed deviations from the observed spectra, but not so important that we could rule out that this oxide is at higher temperature than the silicates. Figure 2 displays for every source the fractional abundance of each dust grain species, ordered by increasing amount of aluminium oxide. Remarkably, melilite appears in all our sources in significant amounts (25-50\%). Only in two sources aluminium oxide is not seen, and there are no sources in which the spectra could be explained with this component alone. Olivine appears in most objects, and its proportion increases as the proportion of aluminium oxide decreases. $\mathrm{Mg}_{0.1} \mathrm{Fe}_{0.9} \mathrm{O}$ and spinel show small amounts, around $10 \%$ and $2 \%$, respectively.

As mentioned in the previous section, the terminal outflow velocity and the mass-loss rate depend on the luminosity and the gas-to-dust mass ratio. The luminosities can be determined from the stellar spectra and the distances listed in Table 2, which have been taken from the Hipparcos catalogue or from $\mathrm{CO}$ shell studies. The gas-to-dust mass ratio, $r_{\mathrm{gd}}$, can be calculated by scaling the terminal velocity obtained with the model (which assumes $r_{\mathrm{gd}}$ equal to 200), to the terminal outflow velocity, $v_{\mathrm{e}}$, observed with the CO lines (Zubko \& Elitzur 2000). The $r_{\mathrm{gd}}$ values derived in this way and their associated massloss rates are listed in Table 2 for each of the CO line velocities given in Table 1, excluding the narrow line components. The average of the gas-to-dust mass ratios (see also Fig. 4) agrees with the value $158 \pm 13$ obtained by Knapp (1985) for the oxygen stars. However, the values of $r_{\mathrm{gd}}$ show a large dispersion, ranging from around 40 in EP Aqr to more than 1000 in V584 Aql. Marengo et al. (1997) found a similar spread in the dust to gas ratios calculated for a set of O-rich sources.

There are several uncertainties involved in this method. Mass-loss rates derived from the $\mathrm{CO}$ measurements show a large spread, even after correcting for the different distances the various authors use. Part of the spread can be understood because the mass-loss rates were derived from different 


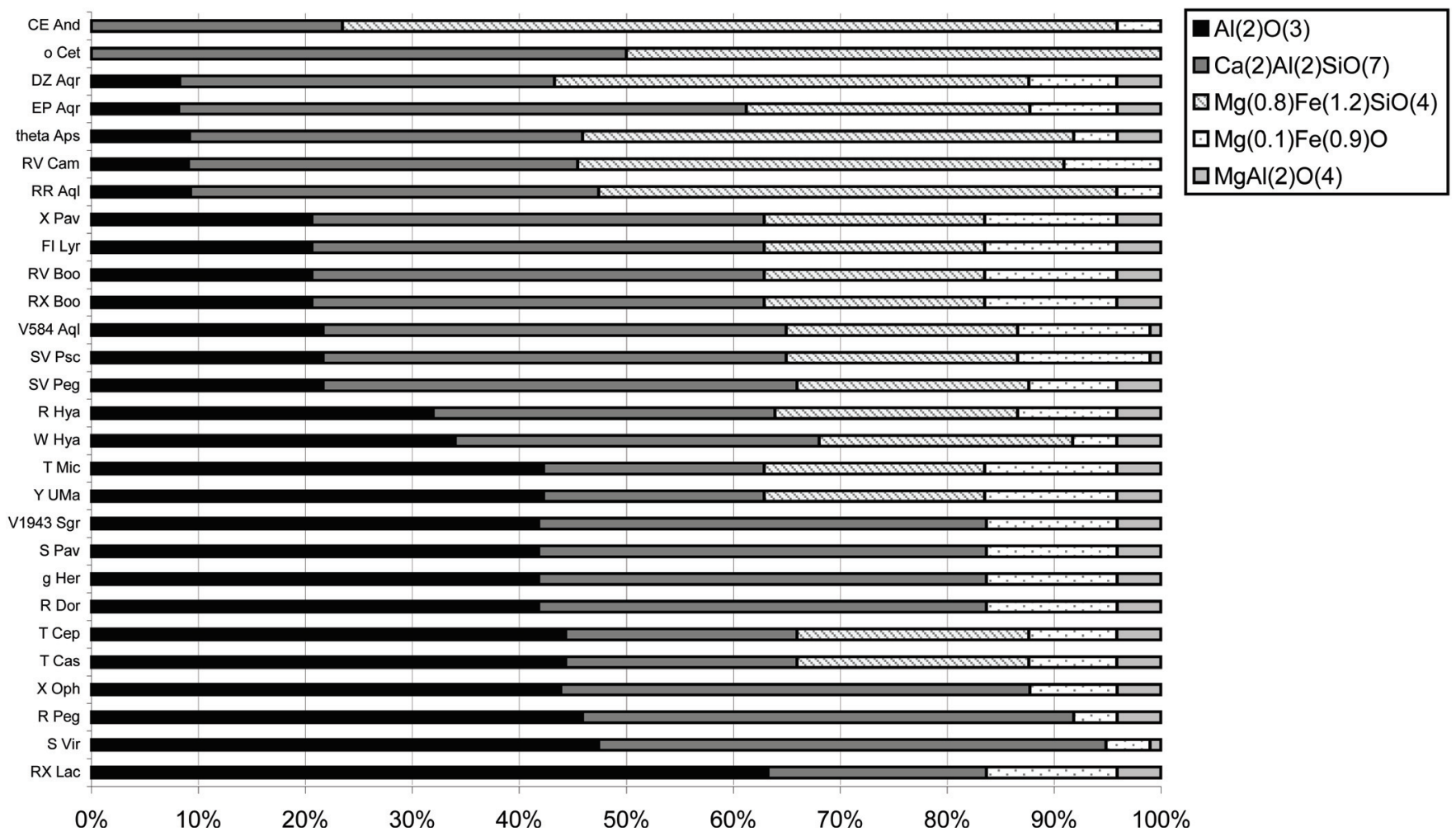

Fig. 2. Fractional abundance of dust species in the CSEs, derived from modelling the SWS spectra.

$\mathrm{CO}$ transitions or combinations of them. These lines probe different parts of the CSE. The higher the CO transition, the closer in the shell it is formed. If the mass-loss rate is not constant then different $\mathrm{CO}$ lines will yield different mass-loss rates. However, the spread in derived values for a single source can be very large (e.g., up to ten times in the case of X Oph). It seems unlikely that such large variations in the mass-loss rate are real. More likely, these differences reflect, in part, the difficulty of deriving mass-loss rates from the very optically thick CO lines. For example, the assumed turbulent velocity can significantly influence their strength (e.g. Kemper et al. 2003).

Moreover, $\mathrm{CO}$ lines often show evidence of double velocity components in the outflow (e.g., SV Psc: Olofsson et al. 2002; Winters et al. 2003), while the derivation of the mass-loss rates is made on the basis of spherical symmetry. This approach is necessitated by the fact that we have no precise information on possible non-spherical dust distributions. Therefore, we cannot link the different components in the $\mathrm{CO}$ lines with components of the dust. This approximation should not introduce any serious errors in our interpretation, because we aim to relate the total dust-driving with the total molecular outflow.

One real concern is stellar variability. All sources in our sample are known pulsating stars, some with large amplitudes. Of course the SWS spectra and the CO measurements where not taken at the same moment or even at the same pulsation phase. The IR spectra of AGB stars are known to vary with pulsation phase (Monnier et al. 1998). These authors find changes in the shape of the $10 \mu \mathrm{m}$ silicate emission depending on phase on top of an IR continuum of variable strength. The shape changes indicate phase-dependent dust formation or dust processing. This is an issue that we cannot address because we do not have IR observations covering different phases. However, the dominant IR variability is the change in strength of IR continuum, which is directly related to the luminosity of the star. Because we use the SWS spectrum both for fixing the stellar photosphere and constraining the dust shell parameters (see Sect. 3) the effect of luminosity variations is minimised.

In spite of the uncertainties described above, dust-driving parameters as derived from the modelling of the SWS spectra are relatively robust. The infrared excess that appears in the observations reflects straightforwardly the momentum transfer from radiation to dust grains, even more so because of the the low opacity in these CSEs. Only a very poor determination of the dust temperatures, which would imply a significantly different wind structure, would seriously affect the reliability of the results.

\section{Discussion}

\subsection{Dynamics of the envelope}

By coupling the velocity expansion values determined from CO observations with the output of DUSTY, we have been able to determine, from the SWS observations, the gas-to-dust ratios and the mass-loss rates in the envelopes of our sources. The question whether the small amount of dust seen in these sources is enough to radiatively drive the wind can now be addressed by comparing these values, $\dot{M}_{\mathrm{SwS}}$, with the mass-loss rates derived from the $\mathrm{CO}$ observations, $\dot{M}_{\mathrm{CO}}$. Agreement between the values calculated from the dust and the CO emissions indicates that the assumptions in the dynamical calculation performed in DUSTY (spherically symmetric, radiatively driven 


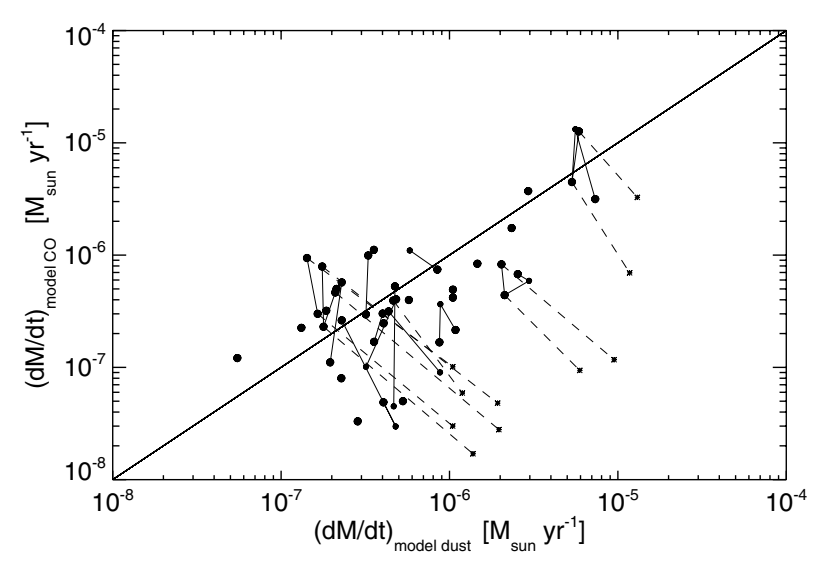

Fig. 3. Comparison of mass-loss rates derived from the SWS observations and from the $\mathrm{CO}$ observations. Solid lines join values that belong to the same object, that have been derived from different CO observations in the literature. The asterisks indicate the mass-loss rate for the narrow CO line component. They are linked by a dashed line to their corresponding $\mathrm{CO}$ broad line value.

wind) are valid. Figure 3 shows the comparison between $\dot{M}_{\text {SwS }}$ and $\dot{M}_{\mathrm{CO}}$ for both the CO broad components (full circles) and the associated narrow line components when they are observed (asterisks, linked by a dashed line). The mass-loss rates for the same object are linked by a solid line. The values of $\dot{M}_{\mathrm{CO}}$ have been scaled to the same distances used to calculate the respective $\dot{M}_{\text {Sws. }}$. As can be seen, the correlation is good for most sources and CO measurements, which leads us to conclude that the dust responsible for the SWS emission bands drives the wind by radiation pressure on the grains. Figure 3 shows that when both a fast and a slow wind are observed, the massloss rates derived by taking the $\mathrm{CO}$ narrow line components are not correlated with $\dot{M}_{\text {Sws }}$. Because we cannot disentangle from our observations which proportion of dust is linked to the slow or to the fast wind, we cannot exclude that the slow wind is also driven by radiation pressure on a small fraction of the dust. However, it is clear that most of the observed dust is involved in the acceleration of the fast wind.

In spite of the good correlation, $\dot{M}_{\text {Sws }}$ and $\dot{M}_{\mathrm{CO}}$ can differ up to a factor of ten. In particular, $\theta$ Aps, V584 Aql, W Hya and $o$ Cet have $\dot{M}_{\text {Sws }}$ higher than $\dot{M}_{\mathrm{CO}}$. This discrepancy could be caused by the existence of more dust around the star than is required to drive the wind. This dust might be distributed in a circumstellar disk or in clumps. Lopez et al. (1997) made a study on the non spherical structures and temporal variations in the dust shell of $o$ Cet. Their observational data were best fitted by a model taking a spherical shell with inhomogeneities or clumps, or by taking thin partial shells with fixed distance between them. The departure from spherical symmetry and homogeneity conditions assumed in the DUSTY code could well explain the discrepancy seen between $\dot{M}_{\mathrm{Sws}}$ and $\dot{M}_{\mathrm{CO}}$ for this star. Another non-exclusive explanation is the variation with time of the mass-loss rate which has been observed in AGB stars (Hawkins 1990; Hashimoto et al. 1998; Monnier et al. 1999). The CO lines used for the determination of $\dot{M}_{\mathrm{CO}}$ trace regions of the envelope at larger radial distances than the mid-infrared dust emission. This implies that if the mass-loss rate was higher in the past, the value of $\dot{M}_{\mathrm{CO}}$ should be higher than the value of $\dot{M}_{\mathrm{SWS}}$, and vice versa. If this is the case, stars for which $\dot{M}_{\mathrm{CO}}$ is greater than $\dot{M}_{\mathrm{SwS}}$, i.e. located above the diagonal line in Fig. 3, would have had previous episodes of higher mass-loss rates. Lower mass-loss rates in the past could explain those cases in which $\dot{M}_{\mathrm{CO}}$ is smaller than $\dot{M}_{\text {Sws }}$. However, since there is no further observational evidence of a sudden increase of the mass-loss rate in AGB stars as a function of time (Habing 1996), the explanation of additional dust present in the envelope but not contributing to the wind (e.g. disks, clumps) is favored for the objects below the diagonal. Interestingly enough, Bergman et al. (2000) produced CO radio line interferometry maps of RV Boo and explained the broad plateau emission observed as caused by a circumstellar disk in Keplerian rotation. In another example of a non-spherical shell of an AGB star, Kahane \& Jura (1996) identified high-velocity CO components in the CSE of X Her, which were symmetrically displaced and that could be interpreted as the red and blue shifted cones of a weakly collimated bipolar outflow.

The well studied case of W Hya is worth to be discussed separately. In addition to CO line measurements, the mass-loss rate of this object has been derived in the literature by different methods. Barlow et al. (1996) and Neufeld et al. (1996) obtained $6 \times 10^{-7} M_{\odot} \mathrm{yr}^{-1}$ and $0.5-3 \times 10^{-5} M_{\odot} \mathrm{yr}^{-1}$ by fitting the thermal water emission observed by ISO-LWS and ISO-SWS, respectively. Zubko \& Elitzur (2000) derived a value of $2.3 \times$ $10^{-6} M_{\odot} \mathrm{yr}^{-1}$ by running the DUSTY code to reproduce a composite spectra of $\mathrm{W}$ Hya from the near-infrared to the millimeter wavelengths. In a recent study, Justtanont et al. (2004) modelled the ISO SWS/LWS spectra and obtained a value of $3.5 \times$ $10^{-8} M_{\odot} \mathrm{yr}^{-1}$. In our study we obtain $8.8-10.9 \times 10^{-7} M_{\odot} \mathrm{yr}^{-1}$, which agrees with the results of Barlow et al. (1996) and Zubko \& Elitzur (2000) within the uncertainties of the modelling, but is quite different from the values of Justtanont et al. (2004) and Neufeld et al. (1996). Hawkins (1990) analyzed W Hya IRAS observations at 60 and $100 \mu \mathrm{m}$ and concluded that this star had a very large shell ( $\sim 1 \mathrm{pc})$, and derived a mass-loss rate of $5 \times 10^{-7} M_{\odot} \mathrm{yr}^{-1}$, similar to our value. Hawkins (1990) discussed that one of the reasons for the large shell of W Hya could be a higher mass-loss rate in the past. From the different mass-loss rates derived by different authors, it is clear that W Hya is more complex than what is supposed in the models, and that the assumptions made have a strong effect on the results. If the extended envelope of $\mathrm{W}$ Hya indicates a higher mass-loss rate in the past, the constant mass-loss approximation may be too simplistic. Moreover, a variable mass-loss rate would lead to different results in studies that use parameters which trace different regions of the shell (e.g. the inner envelope in the near-infrared, outer regions in the far-infrared).

\subsection{Dust composition}

The fact that the dust in the envelopes of our study is mainly composed of aluminium oxide, melilite and olivine, is consistent with the silicate thermodynamic condensation sequence being the responsible mechanism for the formation of the dust. However, without a self-consistent model and further support 


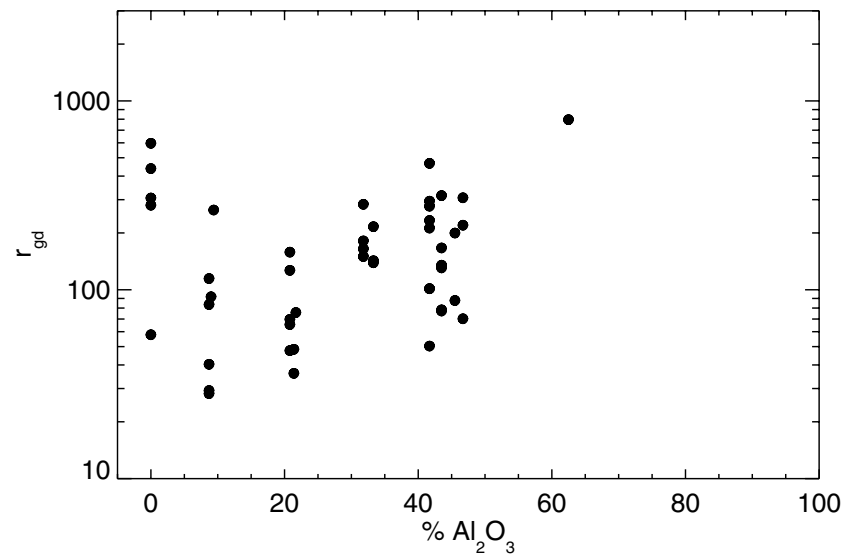

Fig. 4. Gas-to-dust mass ratios as a function of the percentage of $\mathrm{Al}_{2} \mathrm{O}_{3}$ in the dust shell.

from laboratory experiments, we cannot explain how the different dust components have formed in such specific fractional abundances.

Melilite is an important component in the dust of the AGB stars in our sample. Speck et al. (2000) identified melilite in a sample of supergiants, but not in AGB stars. The discrepancy with our results is probably related to the more restricted wavelength range of Speck et al. (2000), 8-13.5 $\mu \mathrm{m}$, which makes the identification of the grain materials more uncertain. In particular, their spectra did not cover the $19 \mu \mathrm{m}$ region where the melilite features make this species distinguishable from other silicates.

We have found further support for the silicate thermodynamic condensation sequence by analyzing the relation between the gas-to-dust mass ratio, $r_{\mathrm{gd}}$, and the fractional abundance of aluminium oxide in the envelope. Figure 4 shows that there is a clear dependency of $r_{\mathrm{gd}}$ on the composition of the dust ( $\theta$ Aps,V584 Aql have been excluded because the disagreement between $\dot{M}_{\text {SWS }}$ and $\dot{M}_{\text {CO }}$ for these stars makes the derivation of $r_{\mathrm{gd}}$ dubious). The higher $r_{\mathrm{gd}}$, the higher the percentage of aluminium oxide in the shell. In those stars in which more dust has condensed in relation with the gas, there is less aluminium oxide and more olivine, that is, dust components that appear in more advance stages in the condensation sequence. Our sample of stars shows therefore various stages of "freeze-out" in the sequence, and that are indicated by the different percentages of aluminium oxide dust. The outlier cluster on the left of Fig. 4 correspond to $o$ Cet. The derived value of $r_{\mathrm{gd}}$ is much higher than in other objects with similar aluminium oxide content. In Sect. 5.1 we have discussed the possibility that the shell of $o$ Cet has extra dust in clumps or multiple shells. Because the model assumes that all dust is involved in driving the wind, the inclusion of the clumped dust increases the terminal wind velocity derived in DUSTY, $v_{\mathrm{e}}$. As a consequence, when we scale this value to the wind velocity from the $\mathrm{CO}$ lines, we obtain a value of $r_{\mathrm{gd}}$ higher than if less dust had been considered.

The correlation between the relative amount of dust in the wind and the presence of "more evolved" dust agrees well with the conclusions of Hron et al. (1997) for the Miras, and extends the validity of the scenario to the stars with lower

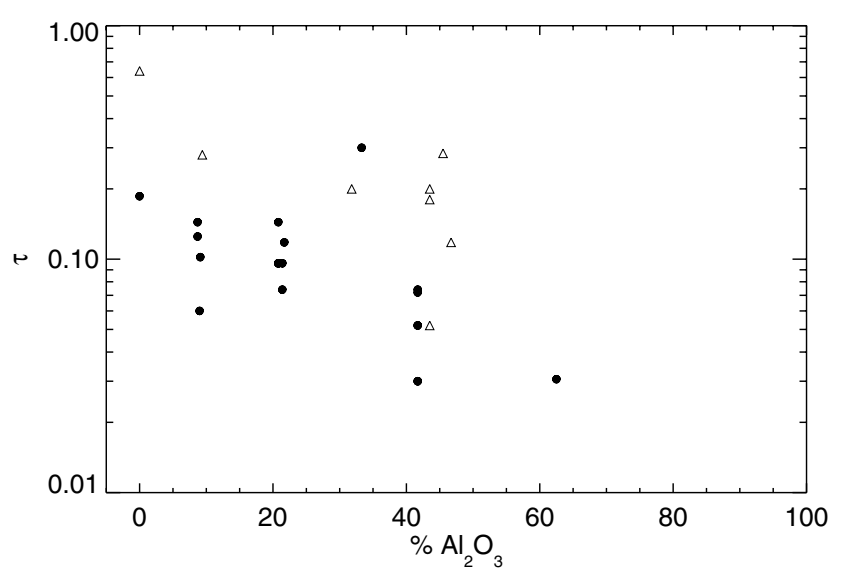

Fig. 5. Dust shell optical depth as a function of percentage of $\mathrm{Al}_{2} \mathrm{O}_{3}$. The Miras are represented by triangles and the semi-regular and long period variables by full circles.

mass-loss rates. However, contrary to these authors, we do not find differences in this trend for the Miras and the semi-regulars (SRs). Figure 5 shows the total amount of dust, represented by the visual optical depth, $\tau$, with respect to the aluminium oxide content, for the Miras (triangles) and the SRs and long period variables (full circles). The correlation between more dust and more evolved dust is also clearly seen here, but it is the same for all stars, although the Miras have higher optical depths, as has been found in other studies (e.g., Hron et al. 1997; Egan \& Sloan 2001). Speck et al. (2000) suggested that, contrary to the Miras, the optical depth in the SRs was lower in those objects showing the narrower silicate features and higher for those with a broader feature. These authors proposed that different mechanisms would control the dust formation in Miras and SRs: the classic condensation sequence could explain the Miras spectral features, while the C/O ratio (Sloan \& Price 1998) would be the controlling agent of dust formation in the SRs. As can bee seen in Fig. 5, we do not find different trends of the optical depth with the aluminium oxide content for the Miras and the SRs, and therefore our study clearly supports a unique controlling mechanism of the dust production in both the Miras and the SRs. Furthermore, we see molecular features of $\mathrm{OH}$ and $\mathrm{H}_{2} \mathrm{O}$ in all the stars of our sample, indicating similar oxygen availability for the Miras and the SRs. Consequently the difference in dust composition between different objects can be better explained by "freeze out" of the condensation sequence rather than by different $\mathrm{C} / \mathrm{O}$ ratios.

In order to further investigate whether there is a correlation between the physical conditions at the inner edge of the shell and the dust composition, we have calculated the gas pressure in this region by assuming an ideal gas law (Gail \& Sedlmayr 1986), and adopting the parameters derived from the model, that is, $\dot{M}_{\mathrm{SWS}}, v_{\mathrm{e}}$ and $T_{\mathrm{d}}$. As can be seen in Fig. 6, there is some indication that the dust composition depends on the conditions at the inner edge. Most of the olivine-rich sources are located in the diagram region of higher temperature and pressure at the inner edge, while the olivine poor sources are mostly found at lower values of these parameters. The three lines in Fig. 6 represent linear fits to the data grouped by their olivine content, 


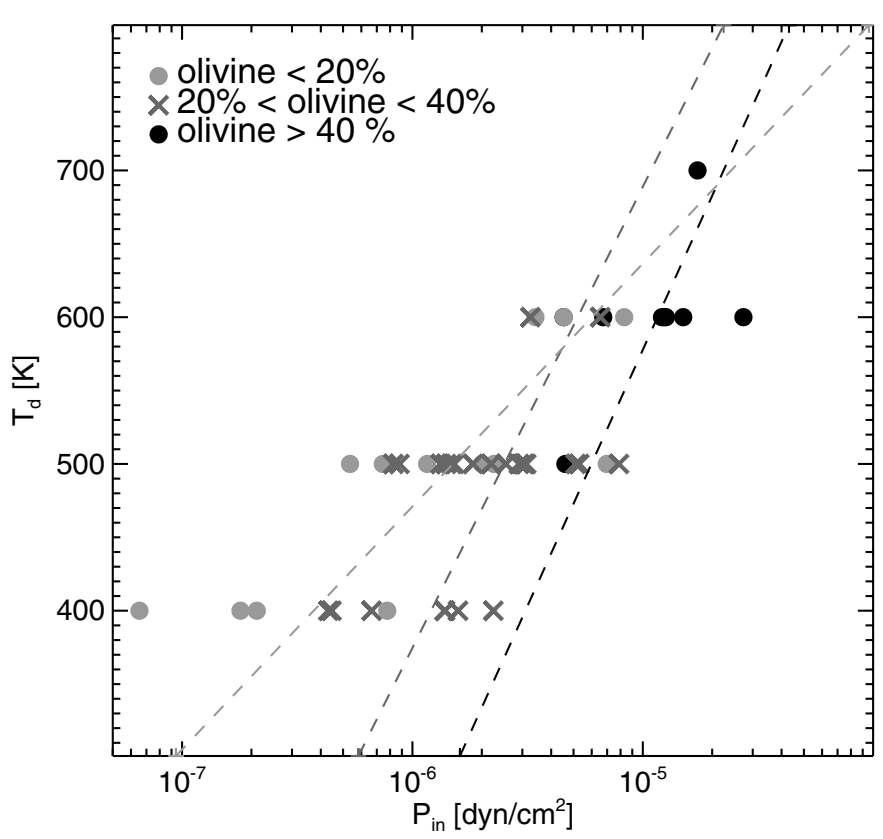

Fig. 6. Pressure and dust temperature at the inner-edge of the dust shell. The sample is split according to the dust composition. The sources with less than 20 percent olivine dust (by mass) are marked with grey circles, between 20 and 40 percent with crosses and above 40 percent with black circles.

marked with the respective colour. Due to the small number of points involved, the slopes of these fits are not significant, however there is a discernible trend of increasing olivine content towards higher pressure and temperature.

The dust composition in the envelopes is consistent with the predictions of the thermodynamic condensation sequence, but the temperature of dust formation is not. As explained above, the dust temperatures at the inner boundary are in the range 400-700 K, well below the theoretical silicate dust formation temperatures. We have already discussed that this temperature is closely related to the silicates, and that we cannot exclude that the aluminium oxide dust is at higher temperature. The low temperatures of the silicates in the envelopes of evolved stars was first noted by Rowan-Robinson \& Harris (1982), and later by Onaka et al. (1989) and Simpson (1991), in studies based on the IRAS LRS data. In a later study using SWS data, Onaka et al. (2002) found a temperature of $700 \pm 100 \mathrm{~K}$ for the inner dust shell temperature of $\mathrm{Z} \mathrm{Cyg}$. Onaka et al. (1989) proposed as an explanation that the main process in silicate formation is mantle growth on pre-existing aluminium grains far out in the circumstellar shell. Otherwise, silicate dust could not be formed, because the density at distances from the star where the temperature is $400 \mathrm{~K}$, is too low for the $\mathrm{SiO}$ molecules to experience enough collisions. Stencel et al. (1990) proposed an alternative explanation, in which the dust formed through the condensation of a chaotic silicate of cosmic composition. By comparing the reduction potentials of $\mathrm{Al}$ and $\mathrm{Si}$, these authors concluded that aluminium metal would reduce silica to produce aluminium oxide plus Si. Only after all $\mathrm{Al}$ had been oxidized, the $\mathrm{Si}$ and $\mathrm{SiO}$ components could begin to oxidize. Sogawa \& Kozasa (1999) developed a model of dust formation in steady state dust-driven winds around oxygen rich AGB stars, and showed that the maximum dust temperatures of the silicate heterogeneous grains (mantle growth on the aluminium oxide core) and of the silicate homogeneous grains were around $400 \mathrm{~K}$ for the lower mass-loss rates $\left(10^{-6} M_{\odot} \mathrm{yr}^{-1}\right)$, which corresponded to a dust formation region located at $10 R_{*}$. These values agree with our results. Further support to low silicate formation temperatures come from laboratory experiments made by Wada et al. (2003), who showed the formation of amorphous silicate at $\sim 650 \mathrm{~K}$, through O-deficient silicate formation.

In addition to silicates and aluminium oxide, we have adopted in our model spinel and $\mathrm{Mg}_{0.1} \mathrm{Fe}_{0.9} \mathrm{O}$. Although spinel has received broad support to explain the $13 \mu \mathrm{m}$ peak (e.g., Posch et al. 1999), its analogue shows a feature around $16.8 \mu \mathrm{m}$ that is not seen in our observational data. Justtanont et al. (2004) reached recently the same conclusion when trying to fit the W Hya spectrum. These facts cast doubts on spinel being responsible for the $13 \mu \mathrm{m}$ feature. In a study of the SWS fullscan spectra of optically thin oxygen-rich circumstellar dust shells, Sloan et al. (2003) ruled out that the $13 \mu \mathrm{m}$ featured was associated with the 16.8 and $32 \mu \mathrm{m}$ features, which has been the main reason for the spinel identification. These authors proposed instead that crystalline forms of $\mathrm{Al}_{2} \mathrm{O}_{3}$ could be the origin of the $13 \mu \mathrm{m}$ peak, as first suggested by Glaccum (1999).

The adoption of $\mathrm{Mg}_{0.1} \mathrm{Fe}_{0.9} \mathrm{O}$ has worked well in reproducing the observed profiles. However, when Posch et al. (2002) proposed this carrier to explain the $19.5 \mu \mathrm{m}$ feature, they assumed a much higher proportion than we have. For example, these authors reproduced approximately a composed spectrum of $g$ Her, V $1943 \mathrm{Sgr}$ and Y UMa by taking $73 \%$ of amorphous alumina and $27 \%$ of $\mathrm{Mg}_{0.1} \mathrm{Fe}_{0.9} \mathrm{O}$, while we only need $10 \%$ of this oxide for $\mathrm{g}$ Her. The discrepancy is due to the inclusion of melilite in our model, because aluminium oxide alone cannot reproduce the observed shape of the $10 \mu \mathrm{m}$ feature. Melilite has also a spectral feature that contributes to the emission around $19 \mu \mathrm{m}$, which implies that less $\mathrm{Mg}_{0.1} \mathrm{Fe}_{0.9} \mathrm{O}$ is required when melilite is considered.

\section{Conclusions}

We have analyzed the ISO-SWS spectra of twenty-eight oxygen-rich AGB stars with optically thin CSEs. Radiative transfer modelling with the code DUSTY has allowed us to determine the composition of the dust and the physical conditions at the dust condensation zone. Through the comparison with $\mathrm{CO}$ observations available in the literature, we have determined the gas-to-dust mass ratios and the mass-loss rates in these sources, and we have studied the wind driving mechanism. Our conclusions are:

1. The amounts of dust present in the envelopes studied, which are characterized by visual optical depths in the $0.03-0.6$ range, are enough to drive the observed wind by radiation pressure on the grains.

2. The comparison between the dust and the CO mass-loss rates shows that some of these sources have circumstellar 
dust that does not contribute to the wind, perhaps located in a disk or clumps.

3. There are indications of variable mass-loss rates in some of the stars.

4. A mixture of aluminium oxide, melilite, olivine, spinel and $\mathrm{Mg}_{0.1} \mathrm{Fe}_{0.9} \mathrm{O}$ fits well the whole range observed spectra. From these species, only melilite is required in proportions greater than $25 \%$ in all cases.

5. The spinel feature at $16.8 \mu \mathrm{m}$ is not seen in any of the SWS spectra of our sample, which casts doubts on the identification of this analogue as the responsible for the $13 \mu \mathrm{m}$ feature.

6. The thermodynamic condensation sequence scenario in which freeze-out occurs due to kinetic effects is supported by: (i) the dust composition; (ii) the aluminium oxide content dependence on the gas-to-dust mass ratio; and (iii) the trend of the olivine content to increase with the pressure and temperature at the shell inner edge. However, the $d e$ rived values of the dust temperatures at the inner edge are substantially lower than those estimated by theory, which supports the findings of previous studies.

Acknowledgements. IA3 is a joint development of the SWS consortium. Contributing institutes are SRON, MPE, KUL and the ESA Astrophysics Division. This research has made use of NASA's Astrophysics Data System Bibliographic Services.

\section{References}

Barlow, M. J., Nguyen-Q-Rieu, Truong-Bach, et al. 1996, A\&A, 315, L241

Barthès, D., Luri, X., Alvarez, R., \& Mennessier, M. O. 1999, A\&AS, 140,55

Begemann, B., Dorschner, J., Henning, T., et al. 1997, ApJ, 476, 199

Bergman, P., Kerschbaum, F., \& Olofsson, H. 2000, A\&A, 353, 257

Cami, J. 2002, Ph.D. Thesis

de Graauw, T., Haser, L. N., Beintema, D. A., et al. 1996, A\&A, 315, L49

Egan, M. P., \& Sloan, G. C. 2001, ApJ, 558, 165

Gail, H.-P. 1990, Rev. Mod. Astron., 3, 156

Gail, H.-P., \& Sedlmayr, E. 1986, A\&A, 166, 225

Glaccum, W. J. 1999, Ph.D. Thesis

Habing, H. J. 1996, A\&AR, 7, 97

Hashimoto, O., Izumiura, H., Kester, D. J. M., \& Bontekoe, T. R. 1998, A\&A, 329, 213

Hawkins, G. W. 1990, A\&A, 229, L5

Hron, J., Aringer, B., \& Kerschbaum, F. 1997, A\&A, 322, 280

Ivezić, v., Nenkova, M., \& Elitzur, M. 1999, User Manual for DUSTY, University of Kentucky Internal Report, accessible at http://www.pa.uky.edu/ moshe/dusty

Jura, M., \& Kahane, C. 1999, ApJ, 521, 302

Justtanont, K., de Jong, T., Tielens, A. G. G. M., Feuchtgruber, H., \& Waters, L. B. F. M. 2004, A\&A, 417, 625
Kahane, C., \& Jura, M. 1996, A\&A, 310, 952

Kemper, F., Stark, R., Justtanont, K., et al. 2003, A\&A, 407, 609

Kholopov, P. N., Samus, N. N., Frolov, M. S., et al. 1998, Combined General Catalogue of Variable Stars, 4.1, Ed (II/214A)

Kim, S., Martin, P. G., \& Hendry, P. D. 1994, ApJ, 422, 164

Knapp, G. R. 1985, ApJ, 293, 273

Knapp, G. R., Young, K., Lee, E., \& Jorissen, A. 1998, ApJS, 117, 209

Kraemer, K. E., Sloan, G. C., Price, S. D., \& Walker, H. J. 2002, ApJS, 140, 389

Lahuis, F., Wieprecht, E., \& Sturm, E. 1997, in ESA SP-419: The first ISO workshop on Analytical Spectroscopy, ed. A. M. Heras, K. Leech, N. R. Trams, \& M. Perry (Noordwijk, The Netherlands), 277

Lodders, K., \& Fegley, B. 1999, in Asymptotic Giant Branch Stars, IAU Symp., 191, 279

Lopez, B., Danchi, W. C., Bester, M., et al. 1997, ApJ, 488, 807

Loup, C., Forveille, T., Omont, A., \& Paul, J. F. 1993, A\&AS, 99, 291

Marengo, M., Canil, G., Silvestro, G., et al. 1997, A\&A, 322, 924

Monnier, J. D., Geballe, T. R., \& Danchi, W. C. 1998, ApJ, 502, 833

Monnier, J. D., Geballe, T. R., \& Danchi, W. C. 1999, ApJ, 521, 261

Mutschke, H., Begemann, B., Dorschner, J., et al. 1998, A\&A, 333, 188

Netzer, N., \& Elitzur, M. 1993, ApJ, 410, 701

Neufeld, D. A., Chen, W., Melnick, G. J., et al. 1996, A\&A, 315, L237

Olofsson, H., González Delgado, D., Kerschbaum, F., \& Schöier, F. L. 2002, A\&A, 391, 1053

Onaka, T., de Jong, T., \& Willems, F. J. 1989, A\&A, 218, 169

Onaka, T., de Jong, T., \& Yamamura, I. 2002, A\&A, 388, 573

Posch, T., Kerschbaum, F., Mutschke, H., Dorschner, J., \& Jäger, C. 2002, A\&A, 393, L7

Posch, T., Kerschbaum, F., Mutschke, H., et al. 1999, A\&A, 352, 609

Rowan-Robinson, M., \& Harris, S. 1982, MNRAS, 200, 197

Salpeter, E. E. 1977, ARA\&A, 15, 267

Sedlmayr, E., \& Dominik, C. 1995, Space Sci. Rev., 73, 211

Sedlmayr, E., \& Krüger, D. 1997, in Astrophysical Implications of the Laboratory Study of Presolar Materials, ed. T. J. Bernatowicz, \& E. Zinner, American Institute of Physics Conference Series (Woodbury, N.Y.), 425

Simpson, J. P. 1991, ApJ, 368, 570

Sloan, G. C., Kraemer, K. E., Goebel, J. H., \& Price, S. D. 2003, ApJ, 594,483

Sloan, G. C., \& Price, S. D. 1998, ApJS, 119, 141

Sogawa, H., \& Kozasa, T. 1999, ApJ, 516, L33

Speck, A. K., Barlow, M. J., Sylvester, R. J., \& Hofmeister, A. M. 2000, A\&AS, 146, 437

Stencel, R. E., Nuth, J. A., Little-Marenin, I. R., \& Little, S. J. 1990, ApJ, 350, L45

Tielens, A. G. G. M. 1990, in From Miras to Planetary Nebulae: Which Path for Stellar Evolution?, 186

Wada, S., Murata, Y., Tokunaga, A. T., \& Watanabe, J. 2003, A\&A, 406, 783

Winters, J. M., Le Bertre, T., Jeong, K. S., Nyman, L.-Å., \& Epchtein, N. 2003, A\&A, 409, 715

Young, K. 1995, ApJ, 445, 872

Zubko, V., \& Elitzur, M. 2000, ApJ, 544, L137 